$14^{\text {th }}$ International Spin Physics Symposium

Osaka, Japan 16-21 October 2000

EDITORS

Kichiji Hatanaka Takashi Nakano Osaka University, Japan

Kenichi Imai Kyoto University, Japan

Hiroyasu Ejiri Osaka University, Japan 


\title{
Polarized Ion Sources For High-Energy Accelerators And Colliders
}

\author{
A.N.Zelenski \\ Brookhaven National Laboratory. Upton. N.Y.. 11973. LSA
}

\begin{abstract}
The recent progress in polarized ion source development is reviewed. In de operation a $1.0 \mathrm{~mA}$ polarized $\mathrm{H}^{\circ}$ ion current is now available from the Optically-Pumped Polarized Ion Source (OPPIS) . In pulsed operation a $10 \mathrm{~mA}$ polarized $\mathrm{H}^{-}$ion current was demonstrated at the TRIL'MF pulsed OPPIS test bench and a $2.5 \mathrm{~mA}$ peak current was obtained from an Atomic Beam Source (ABS) at the INR Moscow test bench. The possibilities for future improvements with both techniques are discussed. A new OPPIS for RHIC spin physics is described. The OPPIS reliably delivered polarized beam for the polarized run at RHIC. The results obtained with in new pulsed ABS injector for the IUCF Cooler Ring are also discussed.
\end{abstract}

\section{INTRODUCTION}

Collider experiments with polarized beams at RHIC /1/ and HERA /2/, will provide essential tests of QCD and the electroweak interaction. Polarization asymmetries and parity violation are the strong signatures for identification of the fundamental processes. which are otherwise inaccessible. Such experiments will require the maximum available luminosity and therefore polarization must be obtained as an extra beam quality without sacrificing intensity. This is already implemented at the electron accelerators. In a storage ring an electron beam is self polarized by the Sokolov-Ternov effect and for linac accelerators a great effort in polarized electron source development was finally rewarded by achievement of $80 \%$ polarization and high beam intensity which will be sufficient to run high-current accelerators like CEBAF at full polarized intensity.

There are a number of proposals to polarize the high-energy proton beam in the storage ring by the Stern-Gerlach effect or spin-filter techniques. But so far the only feasible option is to accelerate the polarized beam produced in the source and make sure that polarization will survive during acceleration and storage. High intensity unpolarized $\mathrm{H}^{-}$ion sources are presently a common choice for high-energy uxcelerators due to the advantage of stripping injection into the accelerator ring. Typical currents for such injectors are in the 20-50 mA range. The present status of high-current polarized ion sources and future prospects for intensity increases to the $20.50 \mathrm{~mA}$ range will be discussed. 


\section{POLARIZATION TECHNIQUES}

In any type of polarized ion source the first step is the generation of an electron-spin polarized atomic beam. The polarization is then transferred to the protons by hyperfine interaction and finally the beam is ionized (a nuclear spin polarized hydrogen beam can be used as a polarized intemal target in ring accelerators or colliders). The difference is in the velocity of the atomic beam. It is comparatively easy to polarize a "slow" (thermal energy) beam by using separating magnets. The advantages of using "fast" (a few keV energy) beams are higher intensity and simple, more efficient ionization (W.Haeberli / 3/).

a). Polarization of "slow" atomic beams: An atomic hydrogen beam is produced in an $R F$ dissociator. A typical beam velocity is about $(1-2) 310^{5} \mathrm{~cm} / \mathrm{s}$, which is achieved by cooling of the dissociator nozzle to a temperature of $30-80 \mathrm{~K}$. The acceptance of separating sextupole magnets is proportional to $\sim \mu \mathrm{B} / \mathrm{kT}$. Field strength is limited to about $1.5 \mathrm{~T}$ by available magnetic materials. The atomic beam of a selected spinstate is directed into the small acceptance of the ionizer (or the storage cell). The initial beam velocity spread and beam scattering in the residual gas along the way (in particular, inside the low vacuum conductance separating magnets) reduce the beam intensity. It appears that everything has already been optimized and a beam intensity of (6-7) $310^{16}$ atoms $/$ can not be significantly improved upon. In pulsed operation, the higher atomic beam intensity of up to $2310^{17}$ atoms/s within a diameter of $15 \mathrm{~mm}$ ionized acceptance was reported. Perhaps, in a transition mode at the front of the beam pulse the scattering is less and the pulsed dissociator works better. The higher field of up to 4-5 $\mathrm{T}$ can be obtained in syperconducting sextupole magnets and a large cold aperture can be used for cryogenics pumping. D.Toporkov has recently built a new polarized deuterium source with high field ( up to $4.8 \mathrm{~T}$ ) superconducting sextupoles and cryogenic pumping. The $\mathrm{D}$ beam intensity is about $50 \%$ higher than in conventional sources $/ 4 /$.

There is a possibility of polarization of hydrogen (deuterium) atoms in spinexchange collisions with optically-pumped alkali-metal atoms in a vapor cell $/ 5 /$. Polarization losses are caused by wall collisions and recombination. A flux of atomic $\mathrm{H}$ in excess of $10^{18}$ atoms/s and an electron polarization of $60 \%$ has been obtained by using a dry-film wall coating to reduce depolarization. Selective ionization must be used to obtain a high nuclear polarization.

In dc atomic beam sources the beam is usually ionized in an ECR-type ionizer and then converted to an $\mathrm{H}^{-}$ion beam in the cesium charge-exchange cell. The total efficiency of the atomic $\mathrm{H}$ beam conversion to the $\mathrm{H}^{-}$ion beam is about $0.1 \%$ and a typical dc polarized $\mathrm{H}^{-} / \mathrm{D}^{-}$ion current is about $10 \mathrm{uA} / 6 \%$. In a pulsed mode the ionization by a $30-50 \mathrm{keV}$ atomic cesium beam is more efficient and up to $40 \mathrm{uA}$ polarized $\mathrm{H}^{-}$ion current was obtained at BNL $/ 7 /$. The ionization efficiency is about $0.5 \%$. In a $\mathrm{D}^{-}$enriched plasma ionizer an order of magnitude higher efficiency was obtained (see A.Belov presentation at this conference).

b). Polarization of "fast" $(0.5-3.0 \mathrm{keV}$ energv) atomic beams: A "fast" atomic $\mathrm{H}$ beam can be easily converted to an $\mathrm{H}^{\prime}$ ion beam just by passing the beam through an 
alkali-metal vapor cell. The $\mathrm{H}^{-}$ion yield is about $9 \%$ in a sodium vapor cell for 0.5 $3.0 \mathrm{keV}$ beam energies and about $16-22 \%$ in a $\mathrm{Rb}$ vapor cell for $0.5-1.0 \mathrm{keV}$ atomic beam energies. The electron -spin polarization of the "fast" $\mathrm{H}$ beam is produced either in a charge-exchange process, when primary protons capture polarized electrons from optically-pumped alkali-metal atoms in a vapor cell, or in spin-exchange collisions $18 /$. This technique is called an "Optically-Pumped Polarized Ion Source" (OPPIS), although polarized electrons can be captured from aferromagnetic foil (as in the original Zavoiski proposal), or from hydrogen, or an alkali-metal atomic beam polarized by separating magnets. The "fast" atomic beam intensity in dc operation is about $10^{17}$ atoms/s (within the ionizer cell's acceptance). This intensity is similar or higher than the "slow" atomic beam intensity in the dc ABS but the ionization efficiency is two orders of magnitude higher. In excess of $1.0 \mathrm{~mA}$ polarized $\mathrm{H}^{-}$ion beam current was obtained in the dc OPPIS $/ 9 /$. In pulsed operation the "fast" beam intensity of $2310^{18}$ atoms/s was obtained within the ionizer acceptance and $10 \mathrm{~mA}$ pulsed polarized $\mathrm{H}^{-}$ion current and $40 \mathrm{~mA}$ of a pulsed polarized proton current was achieved. The feasibility of a $30 \mathrm{~mA}$ polarized $\mathrm{H}^{-}$ion current in a pulsed OPPIS was demonstrated $/ 10 /$.

\section{PULSED ATOMIC BEAM SOURCES AT INR MOSCOW AND IUCF}

Conventional $\mathrm{dc}$ atomic beam sources have been reviewed in detail everywhere 13.6/. A new ABS for the Muenchen tandem accelerator was just completed and a polarized $\mathrm{H}^{-}$ion current of about $10 \mu \mathrm{A}$ was obtained (see R. Hertenberger presentation at this conference). Significant progress since the 1999 spin conference in Protvin has been achieved in pulsed ABS development. A second ABS with a resonant charge-exchange ionizer, based on original INR Moscow design. was built and put into operation at IUCF in 1999. A new source delivers polarized $\mathrm{H}^{-} / \mathrm{D}^{-}$ion beams for injection to the Cooler Injection Synchrotron, which has replaced the IUCF cyclotron as an injector for the Cooler Ring (see A.Belov, V.Derenchuk presentation at this conference). Similar to the INR ABS, a small volume dissociator, fast gas puising and pulsed RF power $(2 \mathrm{~kW}$ ) were used. In a short pulse, an atomic beam intensity of about $2310^{17}$ was obtained within a $15 \mathrm{~mm}$ diameter ionizer acceptance. It is 3 times higher than for similar geometry ABS's which operate in the dc mode.

The plasma injector and extraction system were built at INR Moscow. Simultaneously with the polarized $\mathrm{H}^{-}$beam, an unpolarized $\mathrm{D}^{-}$ion beam of an order of magnitude higher intensity was produced in this source. A very high $D^{\circ}$ ion beam current of $40 \mathrm{~mA}$ was obtained recently at INR Moscow as a result of the plasma ionizer $\mathrm{D}^{+}$to $\mathrm{D}^{-}$converter optimization (at IUCF a $20-30 \mathrm{~mA}$ unpolarized $\mathrm{D}^{-}$current is used routinely for the unpolarized beam physics program). So far the peak polarized beam intensity scales linearly with the unpolarized ion beam current and an $\mathrm{H}^{-}$ion peak intensity of $2.5 \mathrm{~mA}$ was obtained at INR (see A.Belov presentation at this conference). Polarization of about $80 \%$ is expected. The IUCF source produces ubout $1.5 \mathrm{~mA}$ (peak current) $\mathrm{H}^{-}$and $\mathrm{D}^{-}$ion current of $80 \%$ polarization. which meets the requirements of the polarized Cooler injector. 
The estimation of the plasma ionizer efficiency in a short pulse mode is ambiguous because in a short 50-100 $\mu$ s pulse the supply of "fresh" atoms is less than the number of atoms which is already "stored" in the volume of a $30 \mathrm{~cm}$ long ionizer cell. The polarized $\mathrm{H}^{-}$ion current drops rather sharply, therefore the efficiency estimation depends strongly on the averaging time. If it is averaged over a $150 \mu \mathrm{s}$ pulse duration, which is approximately the polarized $\mathrm{H}$ atoms replacement time in the volume of the ionizer cell, then an efficiency of about 6-8\% can be deduced. This result exceeds the initial expectation, which was based on an assumption that the limiting factor would be $\mathrm{H}$ ion stripping by plasma electrons. Precautions were taken to reduce the electron density and there are speculations that a pure $D^{+} D^{-}$plasma is actually produced in the ionizer. At the estimated plasma temperature of about $10 \mathrm{EV}$. the charge-exchange production cross-section $\left(\mathrm{H}^{0}+\mathrm{D}^{-}\right.$to $\left.\mathrm{H}^{-}+\mathrm{D}^{0}\right)$ is about $4310^{-15}$ $\mathrm{cm}^{2}$, and neutralization cross-section $\left(\mathrm{H}^{-}+\mathrm{D}^{+}\right.$to $\left.\mathrm{H}^{0}+\mathrm{D}^{0}\right)$ is about $15310^{-15} \mathrm{~cm}^{2}$, therefore, up to $20 \%$ of the polarized atoms in the cell can be converted to $\mathrm{H}$ ions. Taking into account that a polarized ion beam is affected strongly by the space-charge of the higher current unpolarized $\mathrm{D}^{-}$ion beam, it might be that the achieved efficiency is already close to its maximum. The use of superconducting separating sextupoles and further dissociator and plasma ionizer optimization might produce a polarized $\mathrm{H}^{-}$ ion beam with a peak current of $10 \mathrm{~mA}$.

\section{OPPIS FOR RHIC SPIN PHYSICS}

The polarization facilities at RHIC will provide $70 \%$ polarized proton-proton collisions at energies up to sqrt(s) $=500 \mathrm{GeV}$ and a luminosity of $2310^{32} / \mathrm{cm}^{2} \mathrm{~s} / 1 /$. This luminosity will be obtained with 57 bunches of polarized proton beam having $2310^{11}$ particles/bunch intensity in each ring. The polarized source must produce in excess of $0.5 \mathrm{~mA} \mathrm{H}$ ion current during a $300 \mu$ s pulse, or current 3 duration $>150 \mathrm{~mA}$ $\mu \mathrm{s}$, within a normalized emittance of less than $2 \mathrm{pi} \mathrm{mm} \mathrm{mrad.} \mathrm{This} \mathrm{current} \mathrm{corresponds}$ to $9310^{11}$ particles/pulse. Assuming 50\% beam losses in the LEBT, RFQ, LINAC, and injection to the AGS Booster, that gives $4.5310^{11}$ polarized protons per booster pulse and finally $2310^{11}$ particles in the RHIC bunch. A $1.6 \mathrm{~mA} \mathrm{DC}$ polarized $\mathrm{H}^{-}$ion current was obtained at the TRIUMF OPPIS during the feasibility studies for the polarized beam in the FNAL Tevatron collider $/ 9 /$. The ECR-type primary proton source used in the TRIUMF OPPIS is similar to the OPPIS which was first constucted at KEK /11\%. Polarized beam is not presently required at KEK, and Y.Mori suggested using this source as an injector for RHIC. The source has been upgraded at TRIUMF to meet the RHIC requirements. After upgrade completion, the OPPIS was moved to BNL for installation at the RHIC injector in October of 1999 and has been used for the RHIC spin comissioning in September of 2000.

a). ECR proton source upgrade: A $28 \mathrm{GHz}$ ECR source is used at TRIUMF vs. 18 $\mathrm{GHz}$ at KEK. In the KEK OPPIS the protons are produced in a $6.4 \mathrm{kG}$ field and extracted at a $25 \mathrm{kG}$ field which is nessesary to obtain high polarization. With the 28 $\mathrm{GHz}$ frequency at TRIUMF the resonance field is $10 \mathrm{kG}$. This gives a factor of $2-3$ current gain, other conditions being similar. for the TRIUMF OPPIS. In DC operation. 
the extraction grids are hot, which prevents $\mathrm{Rb}$ metal deposition and provides reliable long-term practically spark-free operation. After modification to $28 \mathrm{GHz} \mathrm{DC}$ operation, a $1.0 \mathrm{~mA} \mathrm{H}$ ion current was obtained with a 199 hole extraction system within the specified emittance (the conventional oven-type sodium ionizer cell was used in initial tests). The same extraction system produced twice as much current in the TRIUMF OPPIS. The difference is partly due to the longer distance between the ECR source and the ionizer (the KEK superconducting solenoid has a room temperature yoke and the TRIUMF solenoid has a cold yoke). In addition, the large hole in the KEK solenoid yoke disturbs the magnetic field symmetry and might be responsible for transverse field components which mis-steer the proton beam. This displacement was observed by direct measurements of the polarized atomic beam profile at the entrance of the ionizer cell. The biggest problem was the degradation of the source performance, within $12 \mathrm{hrs,}$, to $50 \%$ or less of the initial current obtained with fresh grids and cavity assembly. Similar effects were observed in the past for the TRIUMF OPPIS operation, but due to an ample excess of current for routine beam production, the initial decrease was not a problem. After systematic tests the explanation has been found in the ECR gas composition. With a fresh source assembly there is water vapor contamination to the hydrogen in the discharge tube. Water is desorbed from the boron-nitride cups which isolate the plasma from the copper cavity walls. As the cavity dries out the ECR current goes through a maximum (in a few hours) and then drops. A controlled water vapor supply was set up, comprising a water reservoir at 0 deg. $C$, a needle valve and bypass pumping. When properly tuned. the $\mathrm{H}^{-}$ion current recovered to its best value and remained stable for hundreds of hours of operation. Another remarkable feature was very quiet ECR operation. Similar behavior was observed with an oxygen admixture to the hydrogen supply. It was speculated that an oxygen admixture helps to activate the wall surface for better electron emission to the ECR plasma, the same as in ECR sources of multiplycharged ions.

b). Pulsed laser development: DC Ti:sapphire lasers were used for optical pumping in both the KEK and TRIUMF sources. Higher laser power is required for the high-current OPPIS which is easier to obtain in a pulsed operation. Optical pumping of the high density $\mathrm{Rb}$ vapor in the RHIC OPPIS is produced by a pulsed flashlamp pumped $\mathrm{Cr}_{\mathrm{r}} 3+$ doped LiSrAl F ( Cr:LiSAF) crystal operating at $795 \mathrm{~nm}$ wavelength $15 \mathrm{~S}^{1 / 2}$ to $5 \mathrm{P} 1 / 2$ transition $\mathrm{D} 1$ line for $\mathrm{Rb}$ atoms ). It is an excellent replacement for the initially used Ti:sapphire crystal, due to much longer upper level lifetime (a $67 \mu$ s for Cr:LiSAF vs. $3.4 \mu$ s for Ti:sapphire). A $400 \mu$ s laser pulse duration was easily obtained with the LiSAF crystal and a pulsed power of up to 1.0 kW. The maximum duration of the Ti:sapphire laser pulse doesn't exceed $200 \mu \mathrm{s}$. The wavelength is tuned by a 3-plate birefringent filter and a $0.5 \mathrm{~mm}$ thick uncoated etalon. A Burleigh WTA-2000 pulsed wavemeter is used for wavelength measurements and on-line monitoring. The laser linewidth is about $8 \mathrm{GHz}$. The Faraday rotation technique is used for polarization measurements. Nearly $100 \% \mathrm{Rb}$ clectron polarization was measured at a Rb cell thickness of $10^{14}$ atoms $/ \mathrm{cm}^{2}$ in a two cm diameter optically-pumped neutralizer cell.

Al BNL the LiSAF laser is installed in a clean room and the laser beam is delivered to the OPPIS entrance window in a $20 \mathrm{~m}$ long beam transport line. Two focusing 
lenses are used to match the laser beam to the $\mathrm{Rb}$ cell diameter. About half of the initial laser power was measured through the $15 \mathrm{~mm}$ diameter collimator at the entrance of the $\mathrm{Rb}$ cell. The LiSAF crystal is cooled by deionized water ( about 10 $\mathrm{MOhm} / \mathrm{cm}$ ) to reduce the crystal dissolution rate. The water circulation and temperature stabilization is produced by a NESLAB HX-75 chiller-circulator. The laser pulse repetition rate was $1 \mathrm{~Hz}$ and flashlamp input energy is about $60 \mathrm{~J}$. The simmer arc current is 0.5 A. Flashlamp life-time (with a $4.0^{\prime \prime}$ arc gap) at these conditions is more than a million pulses. Laser operation was reliable during several months of OPPIS tests and polarized run in AGS and RHIC.

c). Sodium-jet ionizer cell: The polarized $\mathrm{H}^{-}$ion beam emittance is mostly determined by the ionizer cell aperture diameter and solenoid magnetic field, due to the well known effect of emittance growth during ionizatioin in a magnetic field. The contribution of the primary neutral $\mathrm{H}$ beam emittance is neglegable. A field of $0.15 \mathrm{~T}$ is required to reduce polarization losses to below $2.5 \%$ during ionization. Therefore. the specification for beam emittance of $2.0 \pi \mathrm{mm}$ mrad gives the limit for the sodium cell aperture diameter of $2.0 \mathrm{~cm}$. The sodium vapor flow and corresponding sodium consumption, deposition and penetration into the low field region is proportional to the cube of the cell diameter in the oven-type cell. The laser beam diameter in the optically-pumped cell has to be larger than the proton beam diameter to ensure high electron polarization of $\mathrm{Rb}$ atoms. The laser beam must pass through the ionizer cell, therefore the larger cell aperture results in a higher polarized ion current.

The neutral atomic beam of $3.0 \mathrm{keV}$ energy enters the ionizer, and $\mathrm{H}^{-}$ions produced in the cell can then be accelerated to $35 \mathrm{keV}$ energy (which is required for injection to the RFQ) by ionizer biasing to $-32.0 \mathrm{kV}$. A large cell aperture is essential for this purpose because the neutral beam, collimated to $2.0 \mathrm{~cm}$ in diameter before the cell, should not touch the biased cell parts, otherwise secondary electron emission will cause sparking. A new jet-type ionizer cell with transverse sodium flow was developed to allow large apertures (see Fig.1).

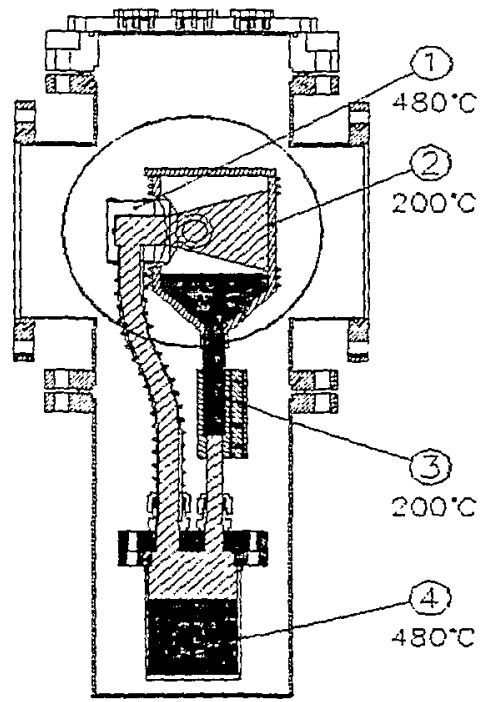

FIGURE 1. Sodjum jet ionizer cell: 1- nozzle: 2- collector: 3- return line 4- sodium seservoir. 
The reservoir is loaded with about $150 \mathrm{~g}$ of sodium metal and heated to $480 \mathrm{deg}$. C. At this temperature the sodium vapor density is about 10 atoms $/ \mathrm{cm}^{3}$. The vapor is delivered through a hot transport tube to the nozzle assembly, which produces a horizontal vapor jet having an effective thickness of about $5310^{15}$ atoms $/ \mathrm{cm}^{2}$, sufficient for $H$ yield saturation. A nozzle slit is $2.0 \mathrm{~mm}$ wide and $20 \mathrm{~mm}$ tall. The transport tube and nozzle temperatures are mainted at $480 \mathrm{deg}$.C. The sodium vapor condenses at the collector walls, which are air-cooled to about 180 deg.C. At this temperature the sodium vapor density is $2310^{12}$ and the sodium viscosity is low. Liquid sodium flows easily down the retum tube and back to the reservoir. The return tube temperature is kept at about 200-250 deg.C by an attached cooling line. The backstream vapor flow through the retum tube is negligible due to the low conductance at 200 deg.C. Sodium in the jet-cell circulates along the path reservoirnozzle-collector-return line-reservoir and the system provides continual. stable operation for hundreds of hours with $150 \mathrm{~g}$ of sodium. Without the circulation the cell works for only 3 hours, measured in a test with the collector water cooled to $25 \mathrm{C}$. The frozen sodium in the collector had a volcano shape perfectly confined within the $10 \mathrm{~cm}$ collector length. The sodium flow outside the cell was much less than with an oven-type cell. The whole ionizer assembly, including the solenoid magnet is electrically isolated from the rest of the OPPIS by $50 \mathrm{~mm}$ thick Delrin flanges.

An immediate acceleration reduces polarized current losses and improves polarization, since the beam energy during spin-transfer collisions can be kept optimal for efficient polarization transfer and ionization (below $3.0 \mathrm{keV}$ ). Sodium losses are greatly reduced in the jet -cell, which is essential because a large diameter is necessary to exclude direct exposure of the biased cell parts to the $3.0 \mathrm{keV}$ energy atomic beam. The atomic beam is reduced to a diameter of $20 \mathrm{~mm}$ by a grounded collimator. The jet-cell apertures are $30 \mathrm{~mm}$ in diameter. The acceleration to $35 \mathrm{keV}$ is produced in a two gap extraction system of $25 \mathrm{~mm}$ and $75 \mathrm{~mm}$ lengths (see Fig. 2). The extraction voltage applied to the first gap is $4.0 \mathrm{kV}$ and to the second gap is $28.0 \mathrm{kV}$.

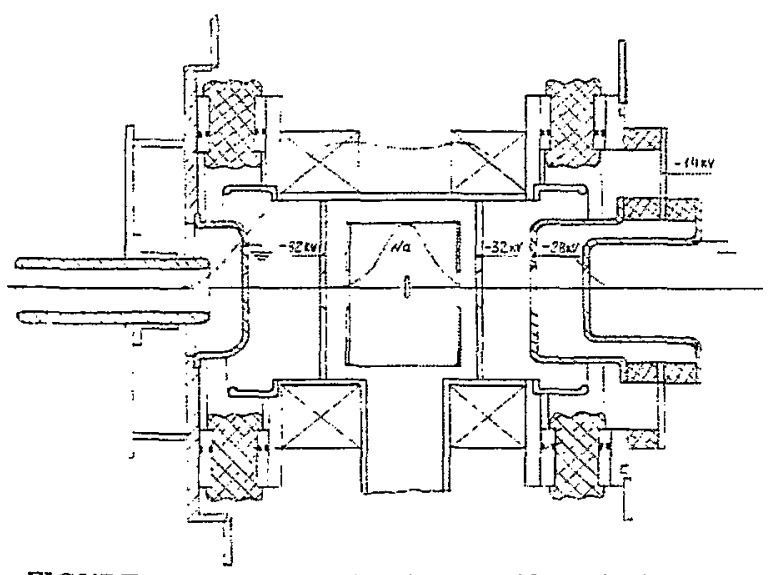

FIGURE 2. H-beam acceleration after Na-jet ionizer cell. 
e).Experimental results: For final tests the BNL OPPIS was installed at the operational TRIUMF OPPIS bench. The TRIUMF Oxford made superconducting solenoid and polarimeters were used in these measurements. A focusing effect of the acceleration gap and improved transport efficiency of the higher energy beam allowed a very high $\mathrm{DC}$ polarized $\mathrm{H}$ ion current of $1.6 \mathrm{~mA}$ to be obtained. This current is similar to the previous record of $1.64 \mathrm{~mA} / 9 /$, but, at this time it was obtained with a 120 hole ECR extraction system instead of a 199 hole, therefore higher polarization was expected. The proton polarization was optimized by use of a Lamb-shift polarimeter and measured after acceleration to $300 \mathrm{keV}$ with a nuclear scattering polarimeter based on the $\mathrm{Li}-6$ ( $\mathrm{p}, \mathrm{He}-3$ ) He-4 reaction (see Fig.3).

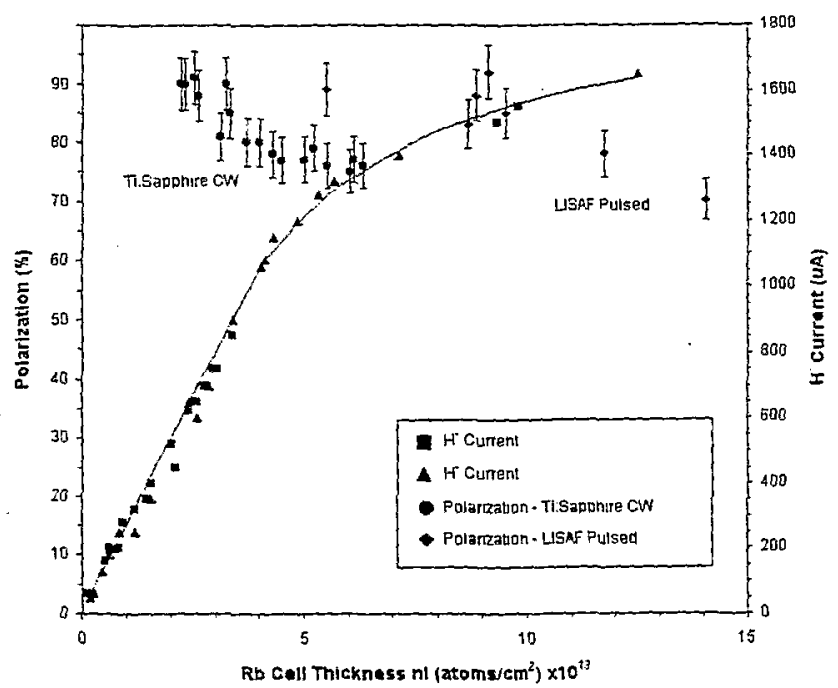

Figure 3: $\mathrm{H}$-current and polarization vs $\mathrm{Rb}$ vapor thickness.

At low $\mathrm{Rb}$ thickness a polarization of $85 \%$ was achieved in DC mode with a single 4.5 W Ti:sapphire laser. At higher Rb density the polarization drops. but the use of a pulsed LiSAF laser restores the polarization to $85 \%$ at $1.6 \mathrm{~mA}$ beam intensity. The polarized source was shipped to $\mathrm{BNL}$ and installed at the $200 \mathrm{MeV}$ linac injector complex in September of 1999. The OPPIS was used for the RHIC SPIN comissioning in August-September 2000. The OPPIS worked continuously and reliably during a three week final run. So far, a 800 uA pulsed polarized $\mathrm{H}^{-}$ion current ha been obtained out of the source and $350 \mathrm{uA}$ was accelerated to $200 \mathrm{MeV}$ for injection to the booster. This gives $6310^{11}$ Hions in a $300 \mu s$ long pulse, which is sufficient to produce the required $2310^{11}$ particles/bunch in RHIC. A 65-72 90 polarization was measured at $200 \mathrm{MeV}$ beam energy after the linac by using a protoncarbon scattering polarimeter. Some polarization losses were obseved in the $35 \mathrm{keV}$ LEBT line due to large spin precession in the bending dipoles and focusing solenoir front of the RFQ. The Lamb-shift polarimeter is presently completed and will be for optimization of the OPPIS polarization at a beam energy of a few $\mathrm{keV}$. 


\section{PULSED OPPIS DEVELOPMENT}

An order of magnitude higher polarized beam intensity will be required for the future polarized RHIC luminosity upgrade and also for the proposed polarized proton facility at the HERA electron-proton collider. The BNL OPPIS is basically a DC source, the pulsed beam is produced only at acceleration after the ionizer cell. the laser for optical pumping is also pulsed. A much higher current of $20-30 \mathrm{~mA}$ was obtained in a pulsed INR, Moscow -type OPPIS with an atomic hydrogen injector in experiments at TRIUMF $/ 10.12$. For polarization measurements, the atomic $\mathrm{H}$ injector was installed at the extended TRIUMF OPPIS test bench, the ECR proton source was replaced with a pulsed He ionizer cell, and a new $45 \mathrm{~cm}$ long Rb cell was installed. The proton polarization was measured by the Lamb-shift polarimeter, which was tested and calibrated with the dc polarized beam from the TRIUMF OPPIS. The source was operated at a $1 \mathrm{~Hz}$ repetition rate and about $100 \mu$ s pulse duration. The optical pumping was produced by the pulsed Cr:LiSAF laser as described above. The TRIUMF superconducting solenoid was designed for ECR source applications. While the total length is $105 \mathrm{~cm}$, the flat field region of a $2.45 \mathrm{~T}$ produced by the main coil is only $30 \mathrm{~cm}$ long. The fields of the other two coils are limited. and to produce a more or less flat field $60 \mathrm{~cm}$ long, only $10.0 \mathrm{kG}$ is achievable. The low field in the optically-pumped $\mathrm{Rb}$ cell causes about $40 \%$ polarization losses. The results of polarization measurements (see Fig.4) are to be compared with the dashed curve which is calculated for polarization in the $10 \mathrm{kG}$ field.

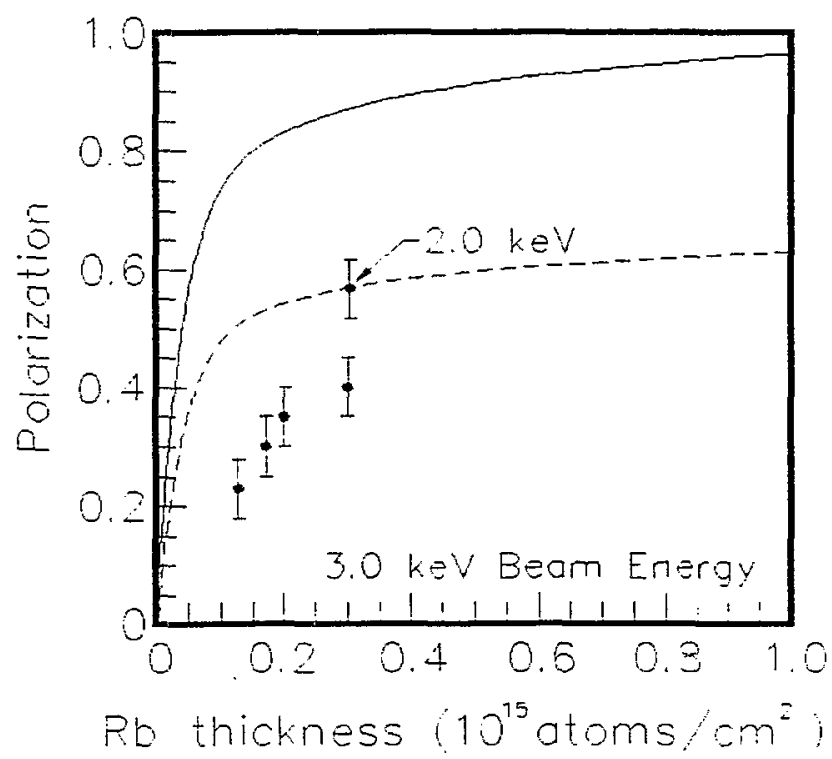

FIGURE 4. Solid line - expected polarization in the $25 \mathrm{kG}$ field. 
The polarized $\mathrm{H}^{-}$ion current is reduced to $8.0 \mathrm{~mA}$ with the He ionizer in operation because of nonhomogeneity of the magnetic field in the cell. The polarized proton current of $50 \mathrm{~mA}$ was obtained by replacement of the sodium ionizer cell by a pulsed He cell. The results of current and polarization measurements are presented in Table.I.

\begin{tabular}{|l|c|c|c|}
\hline TABLE 1. Results of Current and Polarization Measurements. \\
\hline Beam energy (keV) & 2.0 & 3.0 & 4.0 \\
\hline Peak Current I (mA) & 0.1 & $0.5-1.0$ & $0.5-1.5$ \\
\hline $\mathrm{H}^{-}$Current (mA) & 5.0 & 8.0 & 14.0 \\
\hline $\mathrm{H}^{*}$ Current (mA) & 16.0 & 50.0 & - \\
\hline Polarization (F) & 5565 & 4265 & 3065 \\
\hline
\end{tabular}

A new superconducting solenoid is being designed at BNL which will produce a $30 \mathrm{kG}$ field with a flat top $80 \mathrm{~cm}$ long. The calculated proton polarization with such a solenoid is $80-85 \%$. The use of the jet-type sodium ionizer cell and immideate beam acceleration should further increase the achivable polarized $\mathrm{H}^{-}$ion current as it was demonstrated in the BNL OPPIS. The expected polarized $\mathrm{H}^{-}$ion current from the pulsed OPPIS is $30-50 \mathrm{~mA}$, which is close to the present operational unpolarized $\mathrm{H}^{-}$ ion sources.

\section{ACKNOWLEDGEMENTS}

I am thankful to A.Belov and V.Derenchuk for information about the status of their developments and to D.Toporkov and E.Steffen for useful discussions on the limitation of the atomic beam intensity. I acknowledge Y.Mori's (KEK) original proposal and further numerous contributions to the BNL OPPIS development. I would like to thank D.Dutto, P.Levy. G.Wight (TRIUMF). J.Alessi, T.Roser,A.Lechrach (BNL), M.Okamura (RIKEN), and V.Klenov, S. Kokhanovski. V.Zoubets (INR, Moscow') for their contributions to the OPPIS development. The pulsed OPPIS development has been partly funded by DESY through the SPIN Collaboration (spokesperson A.Krish) and by INR, Moscow.

\section{REFERENCES}

1. J.Bunce et al., "Polarized protons at RHIC". Particle World, 3, p.1, (1992).

2. "Prospects of the Spin Physics at HERA". DESY Report 200-95, (1995).

3. W.Haeberli, "Sources of polarized negative ions", Lausanne, p.199,(1990).

4. D.Toporkov, talk given at this conference.

5. J.Fedchat et al., "The Argonne laser-driven polarized D target", AIP Conf.Proc.421, p.129, (1997).

6. T.B. Clegg, "Review of high intensity polarized ion sources", Ref.5, p.336.

7. J.Alessi et al.. "Polarized ion sources for AGS", Helvetica Physica Acta, v.59, p.563, (1986).

8. A.Zelenski, "Optically-pumped polarized ion sources", Int. Workshop on Polarized beams and

targets", Cologne 1995. World Scientific, Singapore, p.111, (1995).

9. A.Zelenski et al., The TRIUMF high-current DC OPPIS", Proc.1995 IEEE PAC, Dallas, p.864, (1995)

10. A.Zelenski et al., "OPPIS development for precision experiments and high-energy colliders", Ref.5, p.372

11. Y.Mori et al., AIP Conf.Proc.117, p.123, (1983)

12. A.Zelenski et al., "OPPIS for RHIC and HERA colliders", DESY Report , 1999. 